\title{
La investigación formativa y su relación con el aprendizaje significativo
}

\author{
Formative research and its relationship with significant learning
}

Pesquisa formativa e sua relação com aprendizagem significativa

\author{
Rosa Kety Cedeño-Intriago ${ }^{\mathrm{I}}$ \\ rosa.cedeno@uleam.edu.ec. \\ Boris Ricardo Tubay-Cedeño II \\ boris.tubay@uleam.edu.ec \\ Darwin Iván Serrano-Zhune III \\ serrranorl.ct@gmail.com
}

\author{
Ketty Carolina Tubay-Cedeño ${ }^{\mathrm{IV}}$ \\ carotubaycedeno1001@gmail.com \\ Roberto Carlos Tubay-Cedeño ${ }^{v}$ \\ robertoctc@hotmail.com
}

Recibido: 11 de mayo de 2019 *Aceptado: 22 de junio de $2019 *$ Publicado: 05 de julio de 2019

\footnotetext{
I. Magíster en Docencia Universitaria de Investigación Educativa, Diploma Superior en Educación Universitaria por Competencias, Licenciado en Servicio Social, Docente de la Universidad Laica Eloy Alfaro de Manabí, Manta, Ecuador.

II. Magíster en Gerencia Educativa, Abogado de los Juzgados y Tribunales de la República, Docente de la Universidad Laica Eloy Alfaro de Manabí, Manta, Ecuador.

III. Especialista en Otorrinolaringología, Médico General, Fuerza Aérea Ecuatoriana, Salinas, Ecuador.

Iv. Médico Cirujano, Hospital Carlos Andrade Marín, Quito, Ecuador.

v. Magíster en Gestión Ambiental, Ingeniero Civil, Universidad Laica Eloy Alfaro de Manabí.
} 


\title{
Resumen
}

El presente artículo basa su argumentación científica en la aplicación de la Teoría del Aprendizaje significativo, misma que utiliza como eje ejecutor la investigación formativa aplicada en el ejercicio de la docencia, haciendo del Estudiante el actor principal del proceso de enseñanza - aprendizaje, privilegiando la investigación-acción, el trabajo académico autónomo, generando ideas, construyendo pensamientos basados en la creatividad, criticidad y dando valor a lo que se estudia.

La metodología utilizada es documental, bibliográfica, webgráfica y explicativa, cuya aplicabilidad permitió estudiar, analizar, interpretar y relacionar la teoría con la praxis educativa áulica, para finalmente concluir en la importancia y aplicabilidad de una cultura investigativa que permita al binomio: Educador - Educando, construir la sociedad del conocimiento significativo.

Palabras clave: Aprendizaje significativo; investigación formativa.

\begin{abstract}
This article bases its scientific argumentation on the application of the Theory of Meaningful Learning, which uses the formative research applied in the exercise of teaching as the executing axis, making the Student the main actor in the teaching-learning process, privileging research action, autonomous academic work, generating ideas, building thoughts based on creativity, criticality and giving value to what is studied.

The methodology used is documentary, bibliographic, webgraphic and explanatory, whose applicability allowed to study, analyze, interpret and relate the theory to the educational praxis praxis, to finally conclude the importance and applicability of a research culture that allows the binomial: Educator - Educating, build the society of meaningful knowledge.
\end{abstract}

Keywords: Significant learning; Formative Research

514 Vol. 5, núm. 3, julio, 2019, pp. 513-527 Rosa Kety Cedeño Intriago, Boris Ricardo Tubay Cedeño, Darwin Iván Serrano Zhune, Ketty Carolina Tubay Cedeño, Roberto Carlos Tubay Cedeño. 


\section{Resumo}

Este artigo baseia sua argumentação científica na aplicação da Teoria da Aprendizagem Significativa, que utiliza a pesquisa formativa aplicada no exercício do ensino como eixo executor, tornando o estudante o ator principal no processo de ensino-aprendizagem, privilegiando a pesquisa -Ação, trabalho acadêmico autônomo, gerando idéias, construindo pensamentos baseados na criatividade, criticidade e valorizando o que é estudado.

A metodologia utilizada é documental, bibliográfica, webgráfica e explicativa, cuja aplicabilidade permitiu estudar, analisar, interpretar e relacionar a teoria a práxis da práxis educacionais, para finalmente concluir a importância e aplicabilidade de uma cultura de pesquisa que permita o binômio: Educador - Educando, construa a sociedade do conhecimento significativo.

Palavras-chave: Aprendizagem significativa; Pesquisa Formativa

\section{Introducción}

David Auzubel, destacado estudioso de la educación, nos dejó como legado científico la Teoría del Aprendizaje Significativo, filosofía educativa que prioriza el cambio social y calidad educativa y cuyo contenido y aplicación aún se mantiene vigente en el accionar académico de docentes que proactivamente inducen al estudiante a ser generadores de su propio cambio cognoscitivo, aplicando la investigación como herramienta que los ínsita a indagar, buscar, descifrar incógnitas, dilucidar dudas, imaginar contextos que los obliga a buscar la forma o actividad mental que los conduzca a concretar científicamente el conocimiento empírico en un conocimiento científico significativo.

Bernardo Restrepo Gómez, PhD. Manifiesta que en la sociedad del conocimiento la calidad de la educación superior está íntimamente asociada con la práctica de la investigación, práctica que se manifiesta de dos maneras: Enseñar a investigar y hacer investigación. La primera hace alusión al ejercicio de la docencia investigativa, esto es, a utilizar la investigación en la docencia, tanto para darle pertinencia científica a ésta, como para familiarizar a los estudiantes con la lógica de la investigación e iniciarlos en su práctica, es decir para adelantar formación investigativa.

515 Vol. 5, núm. 3, julio, 2019, pp. 513-527

Rosa Kety Cedeño Intriago, Boris Ricardo Tubay Cedeño, Darwin Iván Serrano Zhune, Ketty Carolina Tubay Cedeño, Roberto Carlos Tubay Cedeño. 
Conforme a lo anterior este documento relaciona la praxis académica investigativa aplicando la Teoría del Aprendizaje Significativo, para que este último término se evidencie en el desempeño del educando en sus exposiciones, redacción, ortografía, sintaxis, creatividad, pensamiento crítico, entre otros aspectos que ejecutados durante todos los años de su formación profesional nos dará como resultado un ente con experticia en el campo de su competencia y poseído de la seguridad y estabilidad emocional en sus actuaciones laborales cotidianas.

\section{Materiales y Métodos}

La Universidad Laica "Eloy Alfaro” de Manabí, (Ecuador, Manabí, Manta), mantiene un modelo educativo basado en el constructivismo como teoría del aprendizaje, que se fundamenta en el hecho de fomentar en el educando la oportunidad psicológica y cognoscitiva de ir forjando a través de la investigación como elemento base abstractivo, la construcción de un conocimiento crítico, reflexivo, analítico e interpretativo que permita al estudiante explicar la situación dada con propiedad y fundamento científico.

De acuerdo a lo manifestado por Bernardo Restrepo Gómez, "La investigación universitaria es un proceso de búsqueda de nuevo conocimiento, proceso caracterizado por la creatividad del acto, por la innovación de ideas, por los métodos rigurosos utilizados, por validación y juicio crítico de pares. A la investigación está unida íntimamente la creatividad, ya que en buena medida los resultados de la investigación son también creación de conocimiento o de tecnología.

La investigación científica debe operar en la universidad no sólo en el ámbito de las disciplinas o ciencias básicas, sean estas naturales, formales o sociales, sino también en el ámbito de las profesiones o carreras; así lo propuso formalmente la Conferencia Mundial sobre Ciencia para el Siglo XXI: Un Nuevo Compromiso, cuando en la conclusión 56 afirma: "Los gobiernos de los países en desarrollo deben ampliar el status de las carreras científicas, técnicas y educativas y hacer esfuerzos específicos para mejorar las condiciones de trabajo, incrementar su capacidad para retener a los científicos y promover nuevas vocaciones en áreas de ciencia y tecnología." (Unesco, 1999).

516 Vol. 5, núm. 3, julio, 2019, pp. 513-527 Rosa Kety Cedeño Intriago, Boris Ricardo Tubay Cedeño, Darwin Iván Serrano Zhune, Ketty Carolina Tubay Cedeño, Roberto Carlos Tubay Cedeño. 
Considero como maestra universitaria, que el reto docente es precisamente la búsqueda de estrategias académicas que promuevan en el educando la cultura de la investigación y en este cometido juega papel fundamental el aprendizaje significativo que obligatoriamente requiere que la dualidad docente-estudiante (actores básicos de la educación), se compenetren y construyan un espacio de formación en donde el Maestro haciendo uso de la motivación como herramienta psicológica y de comunicación áulica, genere en el Estudiante el deseo de búsqueda, indagación, creatividad y responsabilidad en la construcción del conocimiento significativo, aplicando estrategias, tales como:

1. De comprensión de textos Literarios y No Literarios,

Entendiéndose por textos literarios, aquellos propios de la literatura, como las novelas, cuentos, poesías, relatos, teatro, ensayos, mitos, etc. Es decir, todos los géneros narrativos, líricos y dramáticos; mientras que los No Literarios, se originan por el deseo de transmitir una información objetiva, carecen de acciones poéticas. Su finalidad no es entretener, sino aportar información basada en la realidad.

2. Escritura de documentos académicos: tareas, ensayos académicos, reseñas, foros.

A través de la escritura de estos documentos académicos, el Estudiante en su formación, adquiere la cultura investigativa, pues para desarrollar cualquiera temática que se trate, se proponen tareas de lectura y revisión de materiales científicos como apoyo al desarrollo del trabajo debidamente orientado por el docente responsable de la cátedra. Cabe indicar que el docente debe tener presente que el propósito de la estrategia indicada es que el educando aprenda a exponer, argumentar, resumir, relacionar y jerarquizar contenidos y debatir lo concerniente a la temática investigada.

3. Producción de información visual: Organizadores gráficos, mapas semánticos, conceptuales y mentales.

La información visual como herramienta informativa, se la concibe como un organizador gráfico, constituido en una representación gráfica de conocimientos, los que representan información relevante sobre una determinada temática, aplicando para ello esquemas, dibujos relacionales que permiten a través de palabras claves o frases cortas, desarrollar cognitivamente la idea mental que

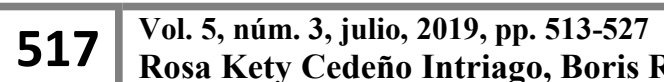

Rosa Kety Cedeño Intriago, Boris Ricardo Tubay Cedeño, Darwin Iván Serrano Zhune, Ketty Carolina Tubay Cedeño, Roberto Carlos Tubay Cedeño. 
se abstrae sobre la temática abordada, rescatando y dando importancia significativa a la capacidad de razonamiento, análisis y síntesis por parte del Estudiante.

\section{Importancia de los organizadores gráficos:}

En la construcción del conocimiento significativo, es importante recalcar lo siguiente:

$>$ El Estudiante se enfoca en la importancia de la temática, resaltando conceptos y vocabulario que son claves y las relaciones entre estos, priorizando la generación del pensamiento crítico y creativo.

$>$ Permite integrar el conocimiento existente con el nuevo.

$>$ Incentiva el desarrollo cognitivo y construcción conceptual.

$>$ El Estudiante se torna en un ente productivo a través de la lectura, la escritura y el pensamiento crítico.

$>$ Favorecen el aprendizaje cooperativo.

$>$ Se basan en criterios de selección y jerarquización, ayudando a los aprendices a aprehender a pensar e innovar el conocimiento.

Ayudan a la comprensión y aprehensión de lo estudiado.

Hay diversos tipos de organizadores visuales de la información, tales como, mapa conceptual, mapa mental, mapa de ideas, mapa semántico, diagrama de ven, diagrama de la espina de pescado, telaraña, uve heurística, línea de tiempo, la cruz categorial, organigramas, diagramas de flujo, árbol de problemas, Mentefactos, entre otros. 


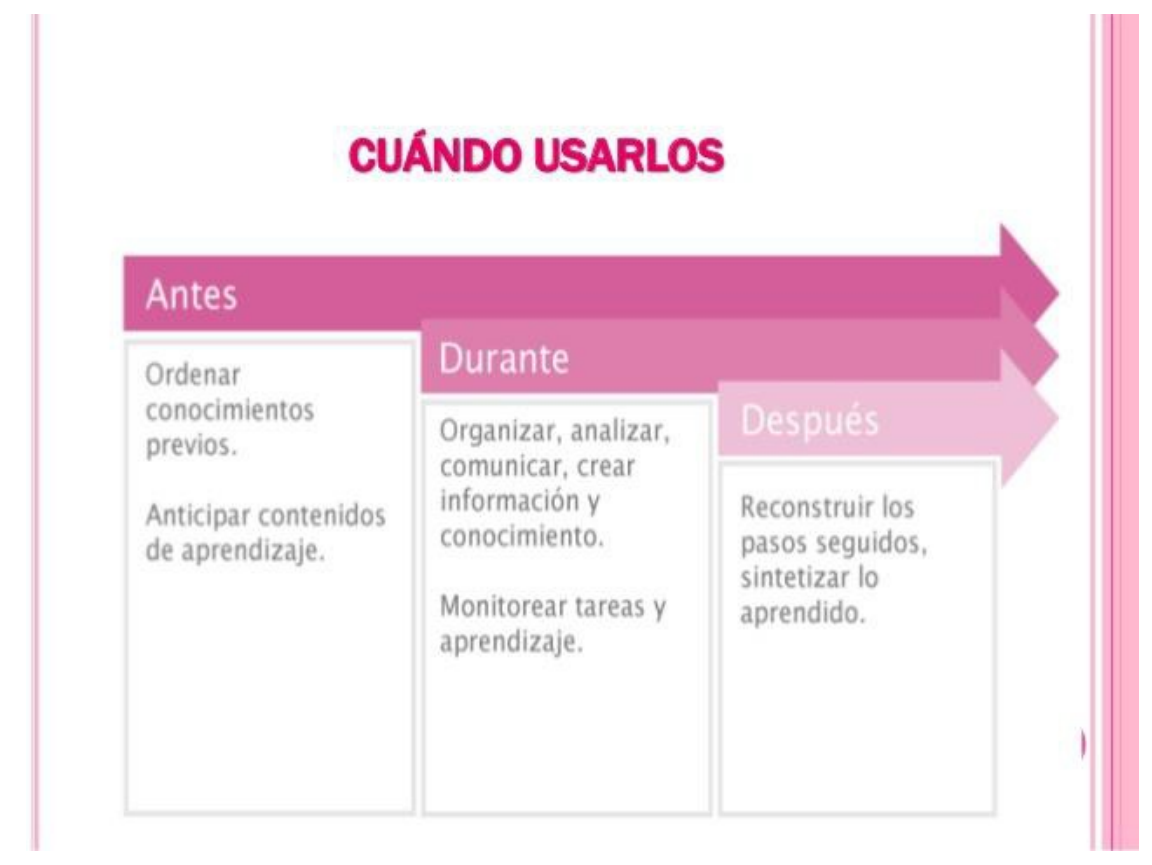

4. Expresión y comunicación de información académica.

El ser humano es un ente eminentemente social por naturaleza, para subsistir necesita como elemento básico comunicarse con su grupo o sociedad. A través de la comunicación aprendemos, sin ella, la vida de las personas carecería de sentido; el poder transmitir información y mensajes nos permite acceder a conocimientos nuevos y enriquecernos como individuo y como cultura.

La competencia de comunicación oral es la capacidad para expresarse mediante el lenguaje hablado y corporal, con el fin de participar y establecerse relaciones en la vida social, académica y profesional, de tal manera que se prioriza la capacidad de transmitir, clara y eficientemente información, pensamientos, opiniones y emociones, tanto de forma oral como escrita, saber escuchar activamente y expresar correctamente la información escrita y oral. En este proceso sobresale la lingüística constituida en la disciplina científica que investiga el origen, la evolución y la estructura del lenguaje, a fin de deducir las leyes que 
rigen las lenguas y las condiciones que hacen posible la comprensión y la comunicación por medio de la lengua natural.

Sobre la base de la lingüística, surge el lenguaje como medio que distingue a un grupo, pueblo o sociedad, mismo que permite al ser humano transmitir sus ideas como fuente básica del arte de comunicarnos a través de la lengua o idioma que le distingue.

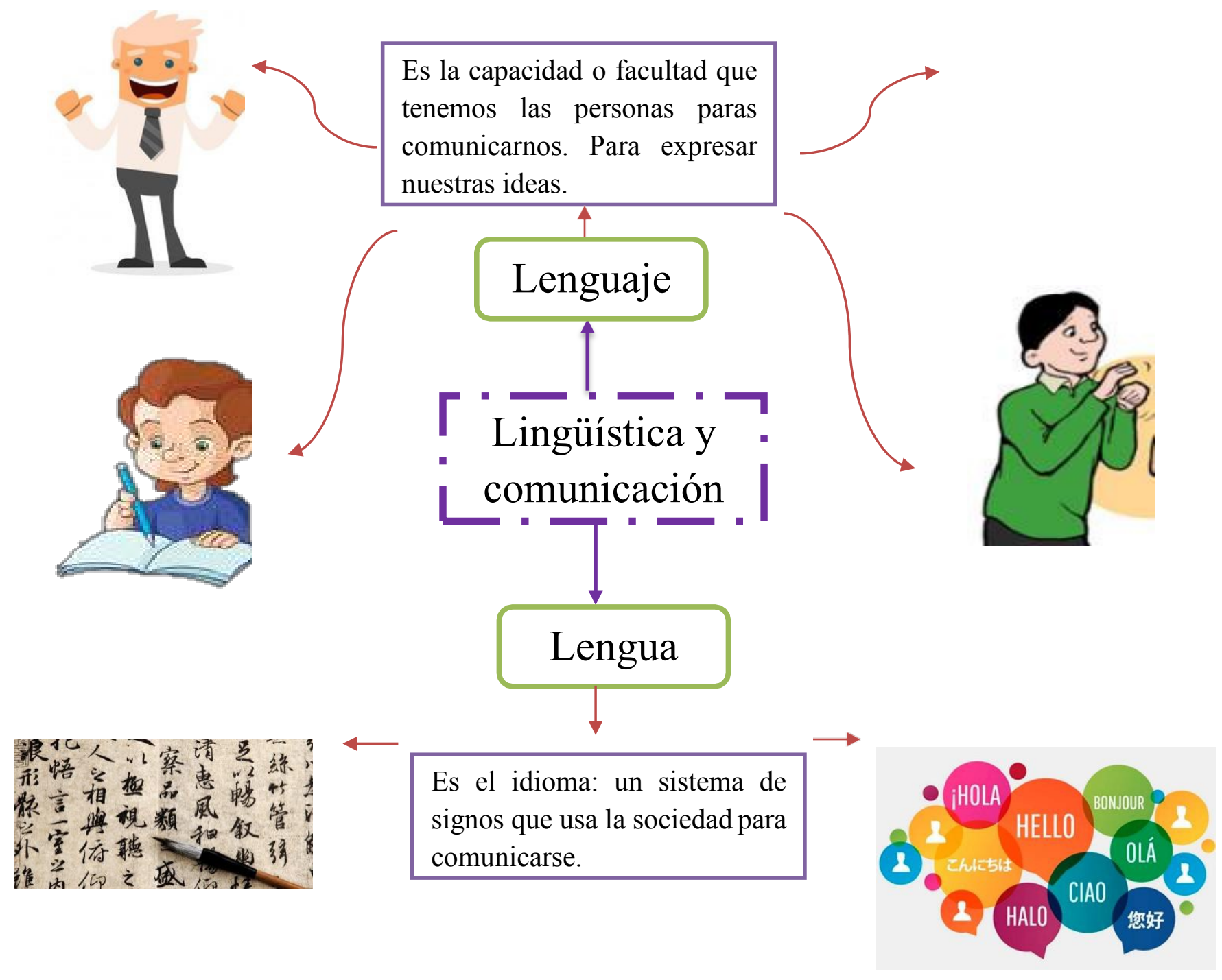




\section{Proceso De La Comunicación}

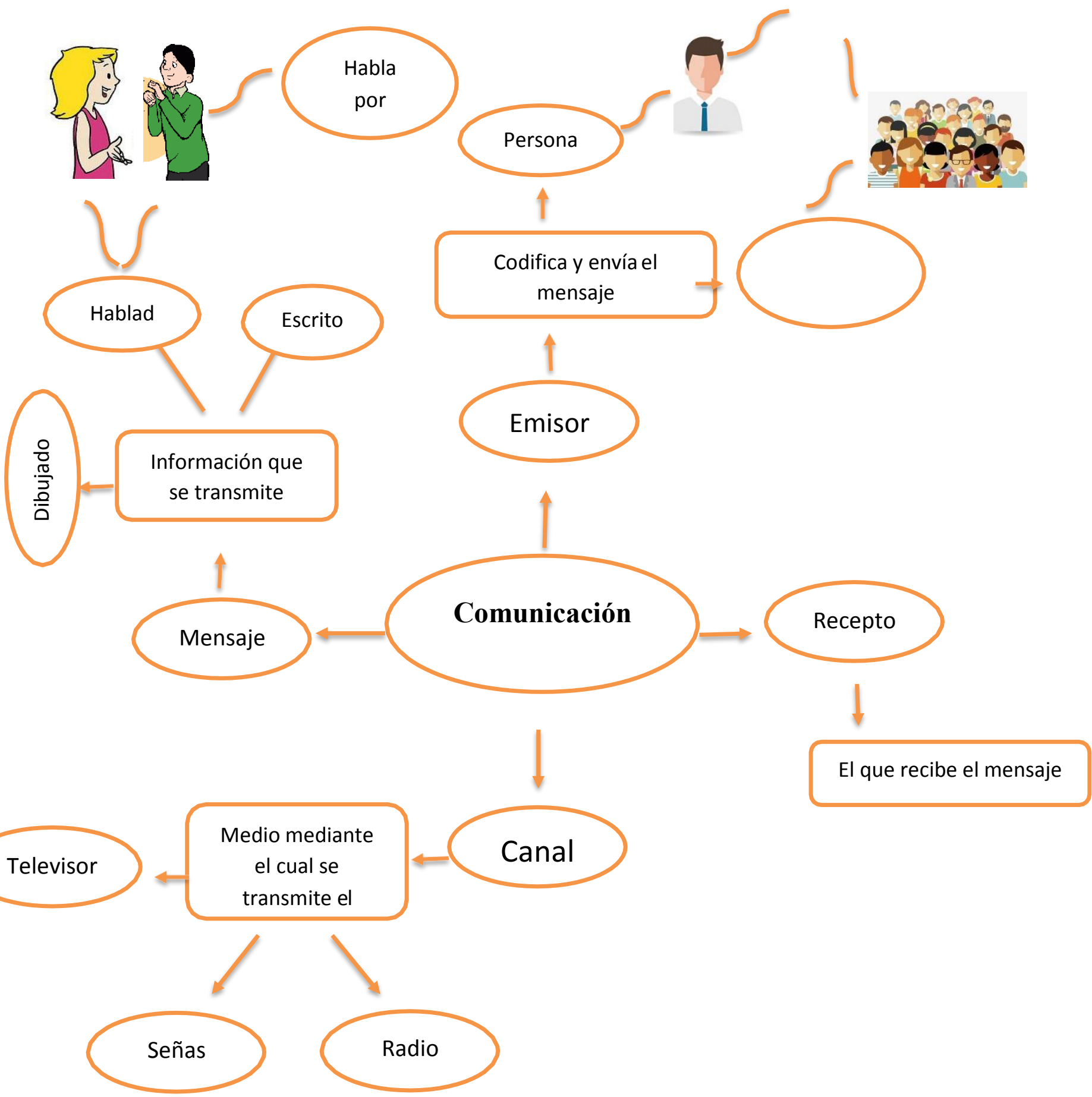
Carolina Tubay Cedeño, Roberto Carlos Tubay Cedeño. 
Según lo establece David Ausubel, psicólogo, cuentista en el campo analítico e interpretativo indica que "el aprendizaje de nuevos conocimientos se basa en lo que ya es conocido con anterioridad. Es decir, la construcción del conocimiento comienza con nuestra observación y registro de acontecimientos y objetos a través de conceptos que ya tenemos; y es precisamente la comunicación como arte y ciencia la que permite y admite la construcción de una red de conceptos que facilitan fortalecer los ya existentes sean empíricos o científicos.

Así, para que este aprendizaje sea posible, hemos de admitir que contamos con un importantísimo vehículo que es el lenguaje: El aprendizaje significativo se logra por intermedio de la verbalización y del lenguaje, requiriendo por tanto de la comunicación entre distintos individuos para consolidar el pensamiento crítico constructivista y generador de un nuevo conocimiento que para que sea significativo debió transitar mentalmente por un proceso de observación, investigación, aprehensión, desarrollo y consolidación.

La teoría del aprendizaje de Ausubel afirma que los nuevos conceptos que deben ser aprendidos, se pueden incorporar a otros conceptos o ideas más inclusivas; estos conceptos o ideas más inclusivas son los organizadores previos; los organizadores previos pueden ser frases o gráficos, en cualquier caso, el organizador avanzado está diseñado para proporcionar lo que llaman los psicólogos cognitivos, el "andamiaje mental", el cual nos conduce a aprehender nueva información, respecto del tema o asunto que nos interesa.

La relación entre docencia e investigación en la educación superior, precisa aclarar la intervención de la investigación científica como eje transversal en la formación del profesional, por tanto está ligada a la necesidad de que la universidad es un centro de formación que responde a la necesidad del entorno social, promoviendo entes que hagan de la investigación científica una herramienta esencial en la construcción de su propio conocimiento y todo ello bajo la tutoría y responsabilidad de un docente que promueva el desarrollo cognitivo y aplique una metodología que incentive al estudiante a sentirse constructor de su propia formación.

522 Vol. 5, núm. 3, julio, 2019, pp. 513-527

Rosa Kety Cedeño Intriago, Boris Ricardo Tubay Cedeño, Darwin Iván Serrano Zhune, Ketty Carolina Tubay Cedeño, Roberto Carlos Tubay Cedeño. 
Ante el comportamiento y responsabilidad docente, debo aclarar, que no se trata de que el Estudiante investigue, construya y exponga sobre una determinada temática, lo que se busca a más de lo anterior es que el profesor universitario estudioso de las distintas teorías del aprendizaje, se constituya en modelo de sapiencia y construcción del conocimiento; en nuestro criterio, que aplique el refuerzo de contingencia áulico que consolida el conocimiento investigado, estudiado, construido, revisado, reforzado y aprobado en el proceso de enseñanza - aprendizaje, sólo en ese momento estaremos frente al aprendizaje significativo e imborrable de nuestra mente.

(Feldman, 2005) Define al aprendizaje como "un proceso de cambio relativamente permanente en el comportamiento de una persona generado por la experiencia.” El aprendizaje significativo es el proceso según el cual se relaciona un nuevo conocimiento o información con la estructura cognitiva del que aprende de forma no arbitraria y sustantiva o no literal. Esa interacción con la estructura cognitiva no se produce considerándola como un todo, sino con aspectos relevantes presentes en la misma, que reciben el nombre de subsumidores o ideas de anclaje (Ausubel, 1976, 2002).

La presencia de ideas, conceptos o proposiciones inclusivas, claras y disponibles en la mente del aprendiz es lo que dota de significado a ese nuevo contenido en interacción con el mismo (Moreira, 2000 a). Pero no se trata de una simple unión, sino que en este proceso los nuevos contenidos adquieren significado para el sujeto produciéndose una transformación de los subsumidores, de su estructura cognitiva, que resultan así progresivamente más diferenciados, elaborados y estables.

Cabe indicar, que aprendizaje significativo no es sólo este proceso, sino que también es su producto. La atribución de significados que se hace con la nueva información es el resultado emergente de la interacción entre los subsumidores claros, estables y relevantes presentes en la estructura cognitiva y esa nueva información o contenido; como consecuencia del mismo, esos subsumidores se ven enriquecidos y modificados, dando lugar a nuevos subsumidores o ideas-ancla más potentes y explicativas que servirán de base para futuros aprendizajes.

523 Vol. 5, núm. 3, julio, 2019, pp. 513-527

Rosa Kety Cedeño Intriago, Boris Ricardo Tubay Cedeño, Darwin Iván Serrano Zhune, Ketty Carolina Tubay Cedeño, Roberto Carlos Tubay Cedeño. 
El aprendizaje implica para el alumno, primero, una necesidad de conocimientos, que tiene lugar en una situación de crisis cognitiva o conflicto cognitivo; este hecho es un contraste por la discrepancia entre los conocimientos previos y los nuevos significados, presentados por el docente, quien estimula el proceso de aprendizaje mediante el planteamiento de retos o problemas para su resolución.

En mayor detalle la Red de Aprendizaje Flipped (FLN) lo define como "un enfoque pedagógico en el que la instrucción directa se mueve desde el espacio de aprendizaje colectivo hacia el espacio de aprendizaje individual y el espacio resultante se transforma en un ambiente de aprendizaje dinámico e interactivo en el que el educador guía a los estudiantes a medida que se aplican los conceptos y puede participar creativamente en la materia"

La Teoría del Aprendizaje Significativo tiene importantes implicaciones psicológicas y pedagógicas; considera que el aprendizaje se construye de manera evolutiva porque se ocupa de lo que ocurre en el aula, postula los principios programáticos para organizar la docencia; es una teoría viva que no sólo se ha limitado a resistir durante tanto tiempo, sino que ha evolucionado a lo largo de su historia, a través de las distintas contribuciones que ha recibido. La aplicación de sus principios a la investigación en educación y a la enseñanza ha permitido, no sólo validar su conocimiento, sino también ampliarlo con interesantes aportaciones que han enriquecido su aplicación y su potencialidad explicativa. Esta teoría pone el énfasis en lo que ocurre en el aula cuando los estudiantes aprenden; en la naturaleza de ese aprendizaje; en las condiciones que se requieren para que éste se produzca; en sus resultados y, consecuentemente, en su evaluación (Ausubel, 1976).

El desarrollo de habilidades, ante todo la transferencia del aprendizaje, o sea, lograr que el aprendizaje anterior, sobre todo el aprendizaje metodológico, sirva para el aprendizaje presente y que éste último potencie aprendizajes futuros (Ausubel, 1983).

524 Vol. 5, núm. 3, julio, 2019, pp. 513-527 Rosa Kety Cedeño Intriago, Boris Ricardo Tubay Cedeño, Darwin Iván Serrano Zhune, Ketty Carolina Tubay Cedeño, Roberto Carlos Tubay Cedeño. 
Habiéndose expuesto fundamentadamente la relación pedagógica entre la construcción del conocimiento y la teoría del aprendizaje significativo, es menester relievar la incidencia de la investigación científica en este cometido académico, considerando que el construir un nuevo conocimiento debe ser indagado siguiendo un proceso estructurado y lógico.

El interés de los docentes por los métodos no expositivos y dentro de éstos por la docencia investigativa, convoca a los profesores universitarios, a conocer y discutir el tema de la relación entre la investigación y la enseñanza, vista desde la pedagogía y desde la misión investigativa de la educación superior. Desde la función pedagógica centramos la atención en la investigación formativa; desde la visión de la misión universitaria de generar conocimiento descriptivo, explicativo y predictivo, y conocimiento sobre la aplicación de conocimiento (conocimiento tecnológico), debiendo por tanto centrar nuestra atención en la investigación científica en sentido estricto. 


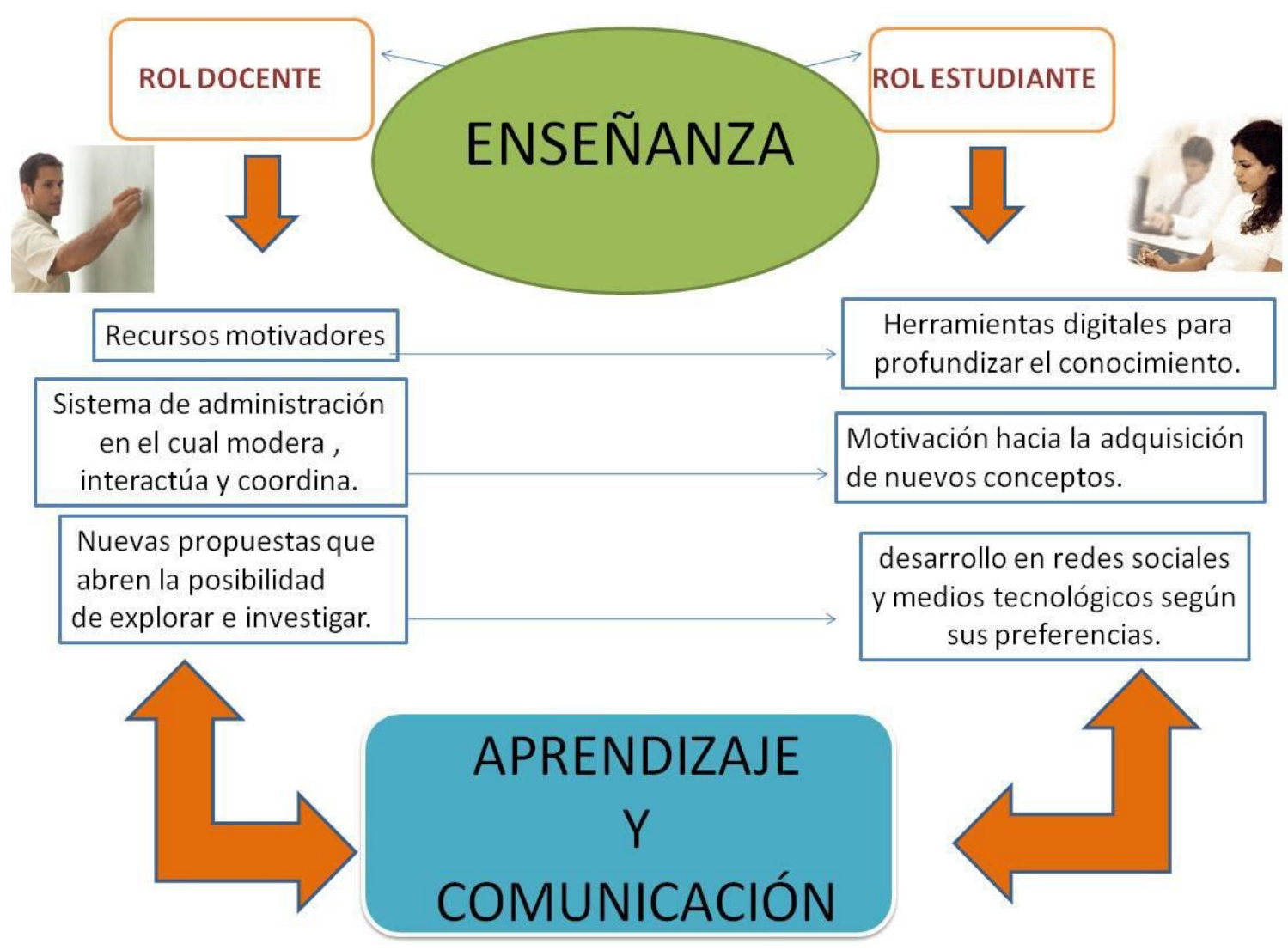

\section{Ilustración 1}

Una de las grandes preocupaciones y expectativas de los estudiantes en todas las disciplinas, es poder relacionar lo aprendido en el aula con su entorno social. El alumno percibe las ideas como si fuera un mundo opuesto en relación a sus experiencias. El aula de clase debe servir como escenario de confrontación de las experiencias y las ideas; debe crear el ejercicio permanente del diálogo y la discusión; debe servir para el encuentro y desencuentro de las propuestas; para el error como punto de referencia continua del aprendizaje; para compartir con el docente y sus compañeros; para generar las interrelaciones al interior de los equipos y entre los equipos. Para construir el sentido compartido de la gestión de comunicaciones y validar el esfuerzo cotidiano de aprehender a aprehender, aprehender haciendo y hacer al aprehender. (Águila, 2005)

\section{6}

Vol. 5, núm. 3, julio, 2019, pp. 513-527

Rosa Kety Cedeño Intriago, Boris Ricardo Tubay Cedeño, Darwin Iván Serrano Zhune, Ketty Carolina Tubay Cedeño, Roberto Carlos Tubay Cedeño. 


\section{Referencias Bibliográficas}

Águila, Y. (2005). La Comunicación en la vida cotidiana. Pontificia Universidad Católica del Perú.

Perú: Asociación de Egresados y Graduados. Obtenido de http://www.pucp.edu.pe/egresados/asocegre/boletinaeg/articulosinteres/44/aguila.pdf> Markarte Markarte . (10 de agosto de 2007).

Ayuso, D y Martínez, V (2006) Evaluación de Calidad de Fuentes y Recursos Digitales: Guías de Buenas Prácticas. Anales de Documentación, No.9, Pág. 17- 42. Universidad de Murcia, España. http://www.um.es/fccd/anales/ad09/ad0902.pdf Tremblay, G. (1995). "La sociedad de la información del Fordismo al Gatesismo", Conferencia Southam presentada en Montreal el 3 de junio de 1995. Universidad

Auzubel, David. (1983). Piscología Educativa. Un punto de vista cognoscitivo. México-Trillas.

BALLESTER V. Antoni. . El aprendizaje significativo en la práctica. España. 2002. Conferencia mundial sobre la ciencia el $1^{\circ}$ de julio 1999 - Texto final.

RESTREPO Gómez, Bernardo. Conceptos y Aplicaciones de la Investigación Formativa, y Criterios para Evaluar la Investigación científica en sentido estricto. Artículo Científico.

527 Vol. 5, núm. 3, julio, 2019, pp. 513-527 